\title{
Search for doubly-charged Higgs bosons at LEP
}

\section{L3 Collaboration}

P. Achard ${ }^{\text {t }}$ O. Adriani ${ }^{\text {q }}$, M. Aguilar-Benitez ${ }^{\mathrm{x}}$, J. Alcaraz ${ }^{\mathrm{x}}$, G. Alemanni ${ }^{\mathrm{y}}$, J. Allaby ${ }^{\mathrm{r}}$, A. Aloisio $^{\text {ab }}$, M.G. Alviggi ${ }^{\text {ab }}$, H. Anderhub ${ }^{\text {at }}$, V.P. Andreev ${ }^{\text {f,ag }}$, F. Anselmo ${ }^{\text {h }}$, A. Arefiev ${ }^{\text {aa }}$, T. Azemoon ${ }^{\text {c }}$, T. Aziz ${ }^{i}$, P. Bagnaia ${ }^{\text {al }}$, A. Bajo ${ }^{\mathrm{x}}$, G. Baksay ${ }^{\mathrm{y}}, \mathrm{L}$. Baksay ${ }^{\mathrm{y}}$, S.V. Baldew ${ }^{\text {b }}$, S. Banerjee ${ }^{\text {i }}$, Sw. Banerjee ${ }^{\text {d }}$, A. Barczyk ${ }^{\text {at,ar }}$, R. Barillère ${ }^{\mathrm{r}}$, P. Bartalini ${ }^{\mathrm{v}}$, M. Basile ${ }^{\text {h }}$, N. Batalova ${ }^{\text {aq }}$, R. Battiston ${ }^{\text {af }}$, A. Bay ${ }^{\mathrm{v}}$, F. Becattini ${ }^{\mathrm{q}}, \mathrm{U}$. Becker ${ }^{\mathrm{m}}$, F. Behner ${ }^{\text {at }}$, L. Bellucci ${ }^{\mathrm{q}}$, R. Berbeco ${ }^{\mathrm{c}}$, J. Berdugo ${ }^{\mathrm{x}}$, P. Berges ${ }^{\mathrm{m}}$, B. Bertucci ${ }^{\text {af }}$, B.L. Betev ${ }^{\text {at }}$, M. Biasini ${ }^{\text {af }}$, M. Biglietti ${ }^{\text {ab }}$, A. Biland ${ }^{\text {at }}$, J.J. Blaising ${ }^{\text {d }}$, S.C. Blyth ${ }^{\text {ah }}$, G.J. Bobbink $^{\text {b }, ~ A . ~ B o ̈ h m ~}{ }^{\text {a }}$, L. Boldizsar ${ }^{1}$, B. Borgia ${ }^{\text {al }}$, S. Bottai ${ }^{\mathrm{q}}$, D. Bourilkov ${ }^{\text {at }}$, M. Bourquin ${ }^{t}$, S. Braccini ${ }^{\text {t, J.G. Branson }}{ }^{\text {an }}$, F. Brochu ${ }^{\text {d }}$, J.D. Burger ${ }^{\mathrm{m}}$, W.J. Burger ${ }^{\text {af }}$, X.D. Cai ${ }^{\mathrm{m}}$, M. Capell ${ }^{\mathrm{m}}$, G. Cara Romeo ${ }^{\mathrm{h}}$, G. Carlino ${ }^{\mathrm{ab}}$, A. Cartacci ${ }^{\mathrm{q}}$, J. Casaus ${ }^{\mathrm{x}}$, F. Cavallari ${ }^{\text {al }}$, N. Cavallo ${ }^{\text {ai }}$, C. Cecchi ${ }^{\text {af }}$, M. Cerrada ${ }^{\mathrm{x}}$, M. Chamizo ${ }^{\mathrm{t}}$, Y.H. Chang ${ }^{\text {av }}$, M. Chemarin ${ }^{\text {w }}$, A. Chen ${ }^{\text {av }}$, G. Chen ${ }^{\mathrm{g}}$, G.M. Chen ${ }^{\mathrm{g}}$, H.F. Chen ${ }^{\mathrm{u}}$, H.S. Chen ${ }^{\mathrm{g}}$, G. Chiefari ${ }^{\mathrm{ab}}$, L. Cifarelli ${ }^{\mathrm{am}}$, F. Cindolo ${ }^{\mathrm{h}}$, I. Clare ${ }^{\mathrm{m}}$, R. Clare $^{\mathrm{ak}}$, G. Coignet $^{\mathrm{d}}$, N. Colino ${ }^{\mathrm{x}}$, S. Costantini ${ }^{\text {al }}$, B. de la Cruz ${ }^{\mathrm{x}}$, S. Cucciarelli ${ }^{\text {af }}$, J.A. van Dalen ${ }^{\text {ad }}$, R. de Asmundis ${ }^{\mathrm{ab}}$, P. Déglon ${ }^{\mathrm{t}}$, J. Debreczeni ${ }^{1}$, A. Degré ${ }^{\mathrm{d}}$, K. Dehmelt ${ }^{\mathrm{y}}$, K. Deiters ${ }^{\mathrm{ar}}$, D. della Volpe ${ }^{\text {ab }}$, E. Delmeire ${ }^{t}$, P. Denes ${ }^{\text {aj }}$, F. DeNotaristefani ${ }^{\text {al }}$, A. De Salvo ${ }^{\text {at }}$, M. Diemoz ${ }^{\text {al }}$, M. Dierckxsens ${ }^{\mathrm{b}}$, C. Dionisi ${ }^{\text {al }}$, M. Dittmar ${ }^{\text {at }}$, A. Doria ${ }^{\text {ab }}$, M.T. Dova ${ }^{\mathrm{j}, 5}$, D. Duchesneau ${ }^{d}$, M. Duda ${ }^{\text {a }}$, B. Echenard ${ }^{\text {t }}$, A. Eline ${ }^{\text {r }}$, A. El Hage ${ }^{\text {a }}$, H. El Mamouni ${ }^{\text {w, }}$ A. Engler ${ }^{\text {ah }}$, F.J. Eppling ${ }^{\mathrm{m}}$, P. Extermann ${ }^{\mathrm{t}}$, M.A. Falagan $^{\mathrm{x}}$, S. Falciano ${ }^{\text {al }}$, A. Favara ${ }^{\text {ae }}$, J. Fay ${ }^{\text {w }}$, O. Fedin ${ }^{\text {ag }}$, M. Felcini ${ }^{\text {at }}$, T. Ferguson ${ }^{\text {ah }}$, H. Fesefeldt ${ }^{\text {a }}$, E. Fiandrini ${ }^{\text {af }}$, J.H. Field $^{\mathrm{t}}$, F. Filthaut ${ }^{\text {ad }}$, P.H. Fisher ${ }^{\mathrm{m}}$, W. Fisher ${ }^{\text {aj }}$, I. Fisk ${ }^{\text {an }}$, G. Forconi ${ }^{\mathrm{m}}$, K. Freudenreich ${ }^{\text {at }}$, C. Furetta ${ }^{z}$, Yu. Galaktionov ${ }^{a a, m}$, S.N. Ganguli ${ }^{i}$, P. Garcia-Abia ${ }^{\mathrm{x}}$, M. Gataullin ${ }^{\text {ae }}$, S. Gentile ${ }^{\text {al }}$, S. Giagu ${ }^{\text {al }}$, Z.F. Gong ${ }^{\mathrm{u}}$, F. Greco ${ }^{\mathrm{ab}}$, G. Grenier ${ }^{\mathrm{w}}$, O. Grimm ${ }^{\text {at }}$, M.W. Gruenewald ${ }^{\mathrm{p}}$, M. Guida ${ }^{\text {am }}$, R. van Gulik ${ }^{\mathrm{b}}$, V.K. Gupta ${ }^{\text {aj }}$, A. Gurtu ${ }^{\mathrm{i}}$, L.J. Gutay ${ }^{\text {aq }}$, D. Haas ${ }^{\text {e }}$, D. Hatzifotiadou ${ }^{\text {h}}$, T. Hebbeker ${ }^{a}$, A. Hervé ${ }^{r}$, J. Hirschfelder ${ }^{\text {ah }}$, H. Hofer ${ }^{\text {at }}$, M. Hohlmann ${ }^{\mathrm{y}}$, G. Holzner ${ }^{\text {at }}$, S.R. Hou ${ }^{\text {av }}$, Y. Hu ${ }^{\text {ad }}$, B.N. Jin ${ }^{\mathrm{g}}$, L.W. Jones ${ }^{\mathrm{c}}$, P. de Jong ${ }^{b}$, I. Josa-Mutuberría ${ }^{x}$, D. Käfer ${ }^{a}$, M. Kaur ${ }^{\text {n }}$, M.N. Kienzle-Focacci ${ }^{t}$, J.K. Kim ${ }^{\text {ap }}$, J. Kirkby ${ }^{\text {r }, ~ W . ~ K i t t e l ~}{ }^{\text {ad }}$, A. Klimentov ${ }^{\text {m,aa }}$, A.C. König ${ }^{\text {ad }}$, M. Kopal ${ }^{\text {aq }}$, V. Koutsenko ${ }^{\text {m,aa }}$, M. Kräber ${ }^{\text {at }}$, R.W. Kraemer ${ }^{\text {ah }}$, A. Krüger ${ }^{\text {as }}$, A. Kunin ${ }^{\text {m}}$, 
P. Ladron de Guevara ${ }^{x}$, I. Laktineh ${ }^{\mathrm{w}}$, G. Landi ${ }^{\mathrm{q}}$, M. Lebeau ${ }^{\mathrm{r}}$, A. Lebedev ${ }^{\mathrm{m}}$, P. Lebrun ${ }^{\text {w }}$, P. Lecomte ${ }^{\text {at }}$, P. Lecoq ${ }^{\mathrm{r}}$, P. Le Coultre ${ }^{\text {at }}$, J.M. Le Goff ${ }^{\mathrm{r}}$, R. Leiste ${ }^{\text {as }}$, M. Levtchenko ${ }^{z}$, P. Levtchenko ${ }^{\text {ag }}$, C. Li ${ }^{\text {u }}$, S. Likhoded ${ }^{\text {as }}$, C.H. Lin ${ }^{\text {av }}$, W.T. Lin ${ }^{\text {av }}$, F.L. Linde ${ }^{\mathrm{b}}$, L. Lista ${ }^{\mathrm{ab}}$, Z.A. Liu ${ }^{\mathrm{g}}$, W. Lohmann ${ }^{\text {as }}$, E. Longo ${ }^{\mathrm{al}}$, Y.S. Lu ${ }^{\mathrm{g}}$, C. $_{\text {Luci }}{ }^{\text {al }}$, L. Luminari $^{\text {al }}$, W. Lustermann ${ }^{\text {at }}$, W.G. Ma ${ }^{\text {u }}$ L. Malgeri ${ }^{t}$, A. Malinin ${ }^{\text {aa }}$, C. Maña ${ }^{x}$, J. Mans ${ }^{\text {aj }}$, J.P. Martin ${ }^{\text {w}}$, F. Marzano ${ }^{\text {al }}$, K. Mazumdar ${ }^{\mathrm{i}}$, R.R. McNeil ${ }^{\mathrm{f}}$, S. Mele ${ }^{\mathrm{r}, \mathrm{ab}}$, L. Merola $^{\text {ab }}$, M. Meschini ${ }^{\text {q }}$, W.J. Metzger ${ }^{\text {ad }}$, A. Mihul ${ }^{\text {k }}$, H. Milcent ${ }^{\mathrm{r}}$, G. Mirabelli ${ }^{\text {al }}$, J. Mnich $^{\text {a }}$, G.B. Mohanty ${ }^{\text {i }}$, G.S. Muanza ${ }^{\text {w }}$, A.J.M. Muijs ${ }^{\text {b }}$, B. Musicar ${ }^{\text {an }}$, M. Musy ${ }^{\text {al }}$, S. Nagy ${ }^{\circ}$, S. Natale ${ }^{\mathrm{t}}, \mathrm{M}$. Napolitano ${ }^{\text {ab }}$, F. Nessi-Tedaldi ${ }^{\text {at }}$, H. Newman ${ }^{\text {ae }}$, A. Nisati ${ }^{\text {al }}$, T. Novak ${ }^{\text {ad }}$, H. Nowak ${ }^{\text {as }}$, R. Ofierzynski ${ }^{\text {at }}$, G. Organtini ${ }^{\text {al }}$, I. Pal ${ }^{\text {aq }}$, C. Palomares ${ }^{\mathrm{x}}$, P. Paolucci ${ }^{\text {ab }}$, R. Paramatti ${ }^{\text {al }}$, G. Passaleva ${ }^{\mathrm{q}}, \mathrm{S}$. Patricelli ${ }^{\text {ab }}$, T. Paul $^{\mathrm{j}}$, M. Pauluzzi $^{\text {af }}$, C. Paus ${ }^{\mathrm{m}}$, F. Pauss ${ }^{\text {at }}$, M. Pedace ${ }^{\text {al }}$, S. Pensotti ${ }^{\mathrm{z}}$, D. Perret-Gallix ${ }^{\mathrm{d}}$, B. Petersen ${ }^{\text {ad }}$, D. Piccolo ab ${ }^{\text {ab }}$ Fierella ${ }^{\text {}}$, M. Pioppi ${ }^{\text {af }}$, P.A. Piroué aj, E. Pistolesi ${ }^{z}$, V. Plyaskin ${ }^{\text {aa }}$, M. Pohl ${ }^{\mathrm{t}}$, V. Pojidaev ${ }^{\mathrm{q}}$, J. Pothier ${ }^{\mathrm{r}}$, D. Prokofiev ${ }^{\mathrm{ag}}$, J. Quartieri ${ }^{\text {am }}$, G. Rahal-Callot ${ }^{\text {at }}$, M.A. Rahaman ${ }^{\mathrm{i}}$, P. Raics ${ }^{\circ}$, N. Raja ${ }^{\mathrm{i}}$, R. Ramelli ${ }^{\text {at }}$, P.G. Rancoita ${ }^{\mathrm{z}}$, R. Ranieri ${ }^{\mathrm{q}}$, A. Raspereza ${ }^{\text {as }}$, P. Razis ${ }^{\text {ac }}$, D. Ren ${ }^{\text {at }}$, M. Rescigno ${ }^{\text {al }}$, S. Reucroft ${ }^{j}$, S. Riemann ${ }^{\text {as }}$, K. Riles ${ }^{\text {c }}$, B.P. Roe ${ }^{c}$, L. Romero ${ }^{\mathrm{x}}$, A. Rosca ${ }^{\text {as }}$, C. Rosenbleck ${ }^{\mathrm{a}}$, S. Rosier-Lees ${ }^{\mathrm{d}}$, S. Roth ${ }^{\text {a }}$, J.A. Rubio ${ }^{\text {r }}$, G. Ruggiero ${ }^{\text {q }}$, H. Rykaczewski ${ }^{\text {at }}$, A. Sakharov ${ }^{\text {at }}$, S. Saremi $^{\text {f }}$, S. Sarkar ${ }^{\text {al }}$, J. Salicio ${ }^{r}$, E. Sanchez ${ }^{\mathrm{x}}$, C. Schäfer ${ }^{\mathrm{r}}$, V. Schegelsky ${ }^{\mathrm{ag}}$, H. Schopper ${ }^{\text {au }}$, D.J. Schotanus ${ }^{\text {ad }}$, C. Sciacca ${ }^{\text {ab }}$, L. Servoli ${ }^{\text {af }}$, S. Shevchenko ${ }^{\text {ae }}$, N. Shivarov ${ }^{\text {ao }}$, V. Shoutko ${ }^{\text {m}}$, E. Shumilov ${ }^{\text {aa }}$, A. Shvorob ${ }^{\text {ae }}$, D. Son ${ }^{\text {ap }}$, C. Souga ${ }^{\text {w }}$, P. Spillantini ${ }^{\text {, }}$, M. Steuer ${ }^{\mathrm{m}}$, D.P. Stickland ${ }^{\text {aj }}$, B. Stoyanov ${ }^{\text {ao }}$, A. Straessner ${ }^{\mathrm{t}}$, K. Sudhakar ${ }^{\mathrm{i}}$, G. Sultanov ${ }^{\text {ao }}$, L.Z. Sun ${ }^{\text {u }, ~ S . ~ S u s h k o v ~}{ }^{a}$, H. Suter ${ }^{\text {at }}$, J.D. Swain ${ }^{j}$, Z. Szillasi ${ }^{\mathrm{y}, 3}$, X.W. Tang ${ }^{\mathrm{g}}$, P. $\operatorname{Tarjan}^{\mathrm{o}}$, L. Tauscher ${ }^{\mathrm{e}}$, L. Taylor $^{\mathrm{j}}$, B. Tellili ${ }^{\mathrm{w}}$, D. Teyssier ${ }^{\mathrm{w}}$, C. Timmermans ${ }^{\text {ad }}$, Samuel C.C. Ting ${ }^{\mathrm{m}}$, S.M. Ting ${ }^{\mathrm{m}}$, S.C. Tonwar ${ }^{\mathrm{i}}$, J.Tóth ${ }^{1}$, C. Tully ${ }^{\text {aj }}$, K.L. Tung ${ }^{\mathrm{g}}$, J. Ulbricht ${ }^{\text {at }}$, E. Valente ${ }^{\text {al }}$, R.T. Van de Walle ${ }^{\text {ad }}$, R. Vasquez ${ }^{\text {aq }}$, V. Veszpremi ${ }^{y}$, G. Vesztergombi ${ }^{1}$, I. Vetlitsky $^{\text {aa }}$, D. Vicinanza ${ }^{\text {am }}$, G. Viertel $^{\text {at }}$, S. Villa ${ }^{\text {ak }}$, M. Vivargent ${ }^{\mathrm{d}}, \mathrm{S}$. Vlachos ${ }^{\mathrm{e}}, \mathrm{I}$. Vodopianov ${ }^{\mathrm{y}}, \mathrm{H}$. Vogel ${ }^{\text {ah }}, \mathrm{H}$. Vogt ${ }^{\text {as }}$, I. Vorobiev ${ }^{\text {ah,aa }}$, A.A. Vorobyov ${ }^{\text {ag }}$, M. Wadhwa ${ }^{\mathrm{e}}$, Q. Wang ${ }^{\text {ad }}$, X.L. Wang ${ }^{\mathrm{u}}$, Z.M. Wang ${ }^{\text {u }}$, M. Weber ${ }^{\text {a }}$, P. Wienemann ${ }^{\text {a }}$, H. Wilkens ${ }^{\text {ad }}$, S. Wynhoff ${ }^{\text {aj }}$, L. Xia ${ }^{\text {ae }}$, Z.Z. Xu ${ }^{u}$, J. Yamamoto ${ }^{c}$, B.Z. Yang ${ }^{u}$, C.G. Yang ${ }^{g}$, H.J. Yang ${ }^{c}$, M. Yang ${ }^{g}$, S.C. Yeh ${ }^{\text {aw }}$, An. Zalite ${ }^{\text {ag }}$, Yu. Zalite ${ }^{\mathrm{ag}}$, Z.P. Zhang ${ }^{\mathrm{u}}$, J. Zhao ${ }^{\mathrm{u}}$, G.Y. Zhu ${ }^{\mathrm{g}}$, R.Y. Zhu ${ }^{\text {ae }}$, H.L. Zhuang ${ }^{\mathrm{g}}$, A. Zichichi ${ }^{\text {h,r, }}$, B. Zimmermann ${ }^{\text {at }}$, M. Zöller ${ }^{\text {a }}$

a III. Physikalisches Institut, RWTH, D-52056 Aachen, Germany ${ }^{1}$

b National Institute for High Energy Physics, NIKHEF, and University of Amsterdam, NL-1009 DB Amsterdam, The Netherlands c University of Michigan, Ann Arbor, MI 48109, USA

${ }^{\mathrm{d}}$ Laboratoire d'Annecy-le-Vieux de Physique des Particules, LAPP, IN2P3-CNRS, BP 110, F-74941 Annecy-le-Vieux cedex, France e Institute of Physics, University of Basel, CH-4056 Basel, Switzerland

${ }^{\mathrm{f}}$ Louisiana State University, Baton Rouge, LA 70803, USA

$\mathrm{g}$ Institute of High Energy Physics, IHEP, 100039 Beijing, China 6 


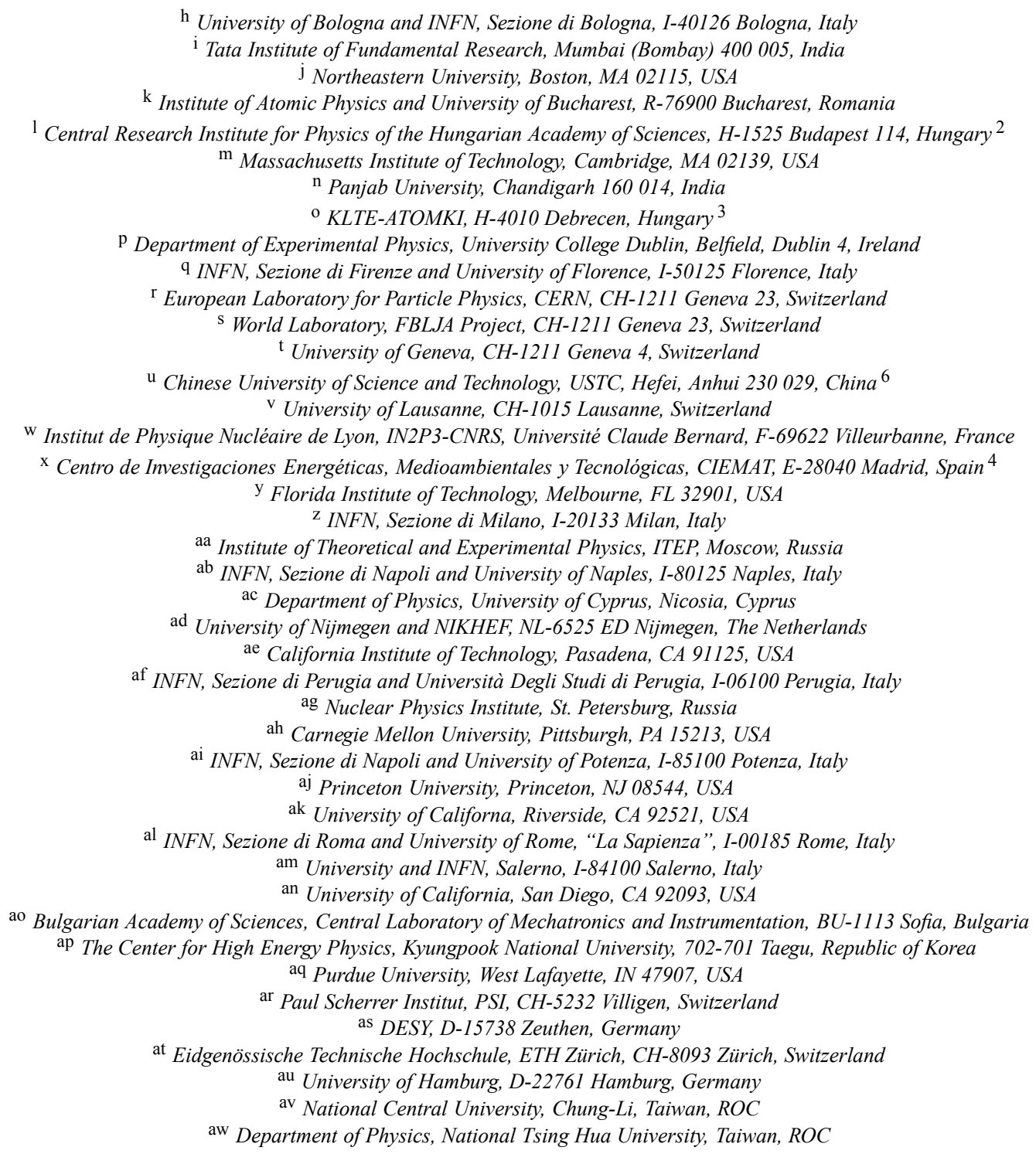

Received 15 September 2003; received in revised form 26 September 2003; accepted 26 September 2003

Editor: L. Rolandi

\begin{abstract}
Doubly-charged Higgs bosons are searched for in $\mathrm{e}^{+} \mathrm{e}^{-}$collision data collected with the L3 detector at LEP at centre-of-mass energies up to $209 \mathrm{GeV}$. Final states with four leptons are analysed to tag the pair-production of doubly-charged Higgs bosons. No significant excess is found and lower limits at 95\% confidence level on the doubly-charged Higgs boson mass are derived. They vary from 95.5 to $100.2 \mathrm{GeV}$, depending on the decay mode. Doubly-charged Higgs bosons which couple to electrons would modify the cross section and forward-backward asymmetry of the $\mathrm{e}^{+} \mathrm{e}^{-} \rightarrow \mathrm{e}^{+} \mathrm{e}^{-}$process. The measurements of these
\end{abstract}


quantities do not deviate from the Standard Model expectations and doubly-charged Higgs bosons with masses up to the order of a $\mathrm{TeV}$ are excluded.

(c) 2003 Published by Elsevier B.V.

\section{Introduction}

In the Standard Model of the electroweak interactions [1] the masses of the fermions and bosons are explained by the Higgs mechanism [2]. A consequence of this mechanism is the existence of an additional particle, the Higgs boson, which, to date, has not been directly observed $[3,4]$. Extensions of the Standard Model predict additional Higgs bosons which can be lighter and hence accessible at current experimental facilities. Among these, doubly-charged Higgs bosons, $\mathrm{H}^{ \pm \pm}$, are expected [5] in several scenarios such as Higgs triplet models, left-right symmetric models and, recently, little Higgs models [6].

Doubly-charged Higgs bosons can be light enough [7] to be directly accessible in $\mathrm{e}^{+} \mathrm{e}^{-}$collisions at LEP through the pair-production mechanism, depicted in Fig. 1(a) and (b). In addition, they can contribute to $\mathrm{e}^{+} \mathrm{e}^{-} \rightarrow \mathrm{e}^{+} \mathrm{e}^{-}$scattering as sketched in Fig. 1(c), producing measurable deviations in the cross section and forward-backward asymmetries for masses of the order of a TeV. This Letter describes the direct search for pair-produced doubly-charged Higgs bosons and the constraints derived from the precision measurement of the $\mathrm{e}^{+} \mathrm{e}^{-} \rightarrow \mathrm{e}^{+} \mathrm{e}^{-}$scattering. Data collected with the L3 detector [8] at centre-of-mass energies, $\sqrt{s}$, up to $209 \mathrm{GeV}$ are used. Results from other LEP experiments were recently reported [9].

The $\mathrm{H}^{ \pm \pm}$couplings to charged leptons are parametrised by the parameters $h_{\ell \ell^{\prime}}$, where $\ell$ and $\ell^{\prime}$ denote the charged lepton flavour. The search for pair-

\footnotetext{
${ }^{1}$ Supported by the German Bundesministerium für Bildung, Wissenschaft, Forschung und Technologie.

2 Supported by the Hungarian OTKA fund under contract Nos. T019181, F023259 and T037350.

3 Also supported by the Hungarian OTKA fund under contract No. T026178.

${ }^{4}$ Supported also by the Comisión Interministerial de Ciencia y Tecnología.

5 Also supported by CONICET and Universidad Nacional de La Plata, CC 67, 1900 La Plata, Argentina.

6 Supported by the National Natural Science Foundation of China.
}

produced doubly-charged Higgs bosons described below assumes $h_{\ell \ell^{\prime}}>10^{-7}$ to ensure that the $\mathrm{H}^{ \pm \pm}$decays before entering the detector and $h_{\mathrm{e} \ell}<10^{-3}$ to suppress large contributions to the cross section from the $t$-channel diagram of Fig. 1(b). The latter assumption corresponds to a conservative estimate of the experimental sensitivities.

Doubly-charged Higgs bosons are conventionally labeled as "left-handed" or "right-handed" [5], referring to different couplings rather than different he-

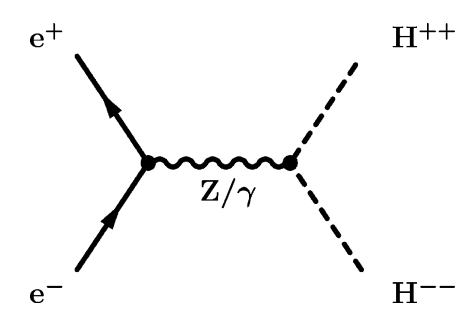

(a)

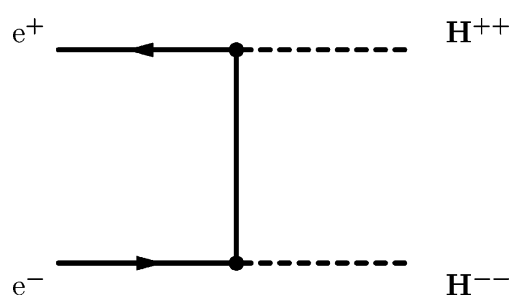

(b)

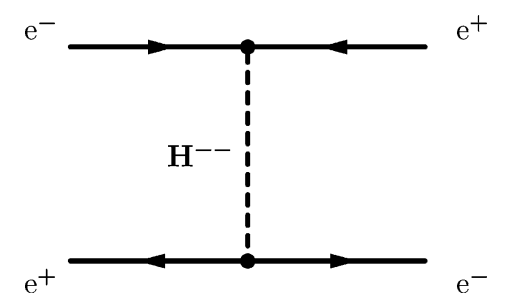

(c)

Fig. 1. (a) $s$-channel and (b) $t$-channel diagrams for the pair-production of doubly-charged Higgs bosons, (c) $u$-channel doubly-charged Higgs boson exchange in the $\mathrm{e}^{+} \mathrm{e}^{-} \rightarrow \mathrm{e}^{+} \mathrm{e}^{-}$ process. 
Table 1

Average centre-of-mass energies and corresponding integrated luminosities

\begin{tabular}{lrrrrrrr}
\hline$\sqrt{s}(\mathrm{GeV})$ & 188.6 & 191.6 & 195.5 & 199.5 & 201.7 & 205.0 & 206.6 \\
\hline Luminosity $\left(\mathrm{pb}^{-1}\right)$ & 176.8 & 29.8 & 84.1 & 84.0 & 39.2 & 80.0 & 130.2 \\
\hline
\end{tabular}

licities. Left-handed $\mathrm{H}^{ \pm \pm}$couple to the $\mathrm{Z}$ boson and the additional $s$-channel diagram results in a pairproduction cross section larger than for right-handed $\mathrm{H}^{ \pm \pm}$. The analysis discussed below concentrates on the latter, less favourable, case. The cross section for the $\mathrm{e}^{+} \mathrm{e}^{-} \rightarrow \mathrm{H}^{++} \mathrm{H}^{--}$process depends $[10,11]$ only on the mass of the doubly-charged Higgs boson, $m_{\mathrm{H}}$, and on $\sqrt{s}$. For $\sqrt{s}=206 \mathrm{GeV}$, it varies from $1 \mathrm{pb}$ for $m_{\mathrm{H}}=60 \mathrm{GeV}$ down to $0.1 \mathrm{pb}$ for $m_{\mathrm{H}}=95 \mathrm{GeV}$.

Pair-production of doubly-charged Higgs bosons produces events with four charged leptons whose flavour depends on the $h_{\ell \ell^{\prime}}$ coupling. In the following, all six possible couplings are considered: $h_{\mathrm{ee}}, h_{\mathrm{e} \mu}$, $h_{\mathrm{e} \tau}, h_{\mu \mu}, h_{\mu \tau}$ and $h_{\tau \tau}$, with the hypothesis that only one coupling at a time is different from zero, which implies that both doubly-charged Higgs bosons in the events have the same decay mode.

If the doubly-charged Higgs boson couples to electrons, it contributes to the differential cross section of the $\mathrm{e}^{+} \mathrm{e}^{-} \rightarrow \mathrm{e}^{+} \mathrm{e}^{-}$process through interference with the additional $u$-channel Feynman diagram depicted in Fig. 1(c). This additional term is calculated [10] to be proportional to

$\frac{h_{\mathrm{ee}}^{2}}{m_{\mathrm{H}}^{2}-u}$,

where $u=-s(1+\cos \theta) / 2$ and $\theta$ is the electron scattering angle. In the following, information on $h_{\text {ee }}$ and $m_{\mathrm{H}}$ is extracted from the comparison of the measured cross section and the forward-backward asymmetry of the $\mathrm{e}^{+} \mathrm{e}^{-} \rightarrow \mathrm{e}^{+} \mathrm{e}^{-}$process with the Standard Model predictions and the doubly-charged Higgs contribution.

\section{Data and Monte Carlo samples}

The search for pair-produced $\mathrm{H}^{ \pm \pm}$uses $624.1 \mathrm{pb}^{-1}$ of data collected at $\sqrt{s}=189-209 \mathrm{GeV}$. Table 1 details the average $\sqrt{s}$ values for the different data taking periods and the corresponding integrated luminosities. Constraints on $\mathrm{H}^{ \pm \pm}$contributions to the $\mathrm{e}^{+} \mathrm{e}^{-} \rightarrow$ $\mathrm{e}^{+} \mathrm{e}^{-}$process are derived from these data and from an additional $66.4 \mathrm{pb}^{-1}$ collected at $\sqrt{s}=130-183 \mathrm{GeV}$.

For the optimisation of the selection and efficiency studies, Monte Carlo events of the process $\mathrm{e}^{+} \mathrm{e}^{-} \rightarrow$ $\mathrm{H}^{++} \mathrm{H}^{--} \rightarrow \ell^{+} \ell^{\prime+} \ell^{-} \ell^{\prime-}$ are generated according to the differential cross sections of Refs. [10,11]. Effects of initial state radiation are included [12] in the generation and final state radiation is modelled with the PHOTOS [13] Monte Carlo. For each $\sqrt{s}$ value listed in Table 1, several $m_{\mathrm{H}}$ points are considered: $m_{\mathrm{H}}=45 \mathrm{GeV}$ and from $m_{\mathrm{H}}=65 \mathrm{GeV}$ up to the kinematic limit $\sqrt{s} / 2$, in steps of $5 \mathrm{GeV}$. For each $m_{\mathrm{H}}$ point, 5000 events are generated for each of the six $h_{\ell \ell^{\prime}}$ couplings. Decays of the tau leptons are described with the TAUOLA [14] Monte Carlo program and JETSET [15] is used to model hadrons produced in these decays.

Standard Model processes are modelled with the following Monte Carlo generators: KK2f [16] for $\mathrm{e}^{+} \mathrm{e}^{-} \rightarrow \mathrm{q} \overline{\mathrm{q}}(\gamma), \mathrm{e}^{+} \mathrm{e}^{-} \rightarrow \mu^{+} \mu^{-}(\gamma)$ and $\mathrm{e}^{+} \mathrm{e}^{-} \rightarrow$ $\tau^{+} \tau^{-}(\gamma)$, BHWIDE [17] for $\mathrm{e}^{+} \mathrm{e}^{-} \rightarrow \mathrm{e}^{+} \mathrm{e}^{-}(\gamma)$, EXCALIBUR [18] for the four-fermion processes $\mathrm{e}^{+} \mathrm{e}^{-} \rightarrow \mathrm{q}^{\prime} e v_{e}, \mathrm{e}^{+} \mathrm{e}^{-} \rightarrow \ell^{+} \ell^{-} \mathrm{q} \overline{\mathrm{q}}$ and $\mathrm{e}^{+} \mathrm{e}^{-} \rightarrow$ $\ell^{+} \ell^{-} \ell^{\prime+} \ell^{\prime-}$, PYTHIA [15] and KORALW [19] for four-fermion final states of the $\mathrm{e}^{+} \mathrm{e}^{-} \rightarrow \mathrm{ZZ}$ and $\mathrm{e}^{+} \mathrm{e}^{-} \rightarrow \mathrm{W}^{+} \mathrm{W}^{-}$processes, respectively, which are not covered by the EXCALIBUR simulations and PHOJET [20] and DIAG36 [21] for hadron and lepton production in two-photon interactions, respectively. The L 3 detector response is simulated using the GEANT program [22] which takes into account the effects of energy loss, multiple scattering and showering in the detector. Time-dependent detector inefficiencies, as monitored during the data taking periods, are included in the simulations.

\section{Search for pair-produced doubly-charged Higgs bosons}

The signature of the $\mathrm{e}^{+} \mathrm{e}^{-} \rightarrow \mathrm{H}^{++} \mathrm{H}^{--} \rightarrow$ $\ell^{+} \ell^{\prime+} \ell^{-} \ell^{\prime-}$ process consists of four leptons, whose flavour depends on the $h_{\ell \ell^{\prime}}$ coupling. For electrons, 
Table 2

Analyses used for the different couplings and the corresponding final states

\begin{tabular}{lll}
\hline Coupling & $\mathrm{H}^{++} \mathrm{H}^{--} \rightarrow$ & Analyses \\
\hline$h_{\mathrm{ee}}$ & $\mathrm{e}^{+} \mathrm{e}^{+} \mathrm{e}^{-} \mathrm{e}^{-}$ & eeee, eee $\gamma$ \\
$h_{\mathrm{e} \mu}$ & $\mathrm{e}^{+} \mu^{+} \mathrm{e}^{-} \mu^{-}$ & ee $\mu \mu, \mathrm{e} \gamma \mu \mu$ \\
$h_{\mathrm{e} \tau}$ & $\mathrm{e}^{+} \tau^{+} \mathrm{e}^{-} \tau^{-}$ & ee $\tau \tau$, ee-jet-jet, e $\gamma-$ jet-jet \\
$h_{\mu \mu}$ & $\mu^{+} \mu^{+} \mu^{-} \mu^{-}$ & $\mu \mu \mu \mu, \mu \mu \mu-\mathrm{MIP}$ \\
$h_{\mu \tau}$ & $\mu^{+} \tau^{+} \mu^{-} \tau^{-}$ & $\mu \mu-$ jet-jet, $\mu \mu \tau \tau$ \\
$h_{\tau \tau}$ & $\tau^{+} \tau^{+} \tau^{-} \tau^{-}$ & ee- $\tau \tau$, ee-jet-jet, e $\gamma-$ jet-jet, $\mu \mu-$ jet-jet, $\mu \mu \tau \tau, \tau \tau \tau \tau$ \\
\hline
\end{tabular}

muons or leptonically decaying tau leptons this signature is clean and little background is expected from lepton pair-production and four-fermion processes. Events with tau leptons which decay into hadrons have a larger background from the four-fermion $\mathrm{e}^{+} \mathrm{e}^{-} \rightarrow$ $\ell^{+} \ell^{-} \mathrm{q} \overline{\mathrm{q}}$ process and from two-photon interactions. The analysis proceeds from the identification of leptons to the preselection of events compatible with the signal signature. Finally, cuts on the lepton energies and global event variables further reduce backgrounds.

Electrons are identified by requiring a well isolated cluster in the electromagnetic calorimeter, formed by at least two adjacent crystals, with an associated track in the tracking chamber. The shower shape of this cluster must be compatible with that of an electromagnetic particle.

Muons are reconstructed by requiring tracks in the muon spectrometer matched with tracks in the central tracker. To reject cosmic background, muon candidates must be in time with the beam crossing.

In addition to their leptonic decays, tau leptons are identified by requiring low-multiplicity jets associated with one, two or three tracks. Narrow and isolated jets are selected by comparing their energy to that deposited in $10^{\circ}$ and $30^{\circ}$ cones around the jet axes.

To increase the selection efficiency, two additional classes of particles are considered: photons, which correspond to electron candidates which fail the track matching criteria, and minimum ionising particles in the calorimeters, MIPs, having an associated track in the central tracker, which tag muons.

Nine analyses are built which rely on the exclusive identification of four leptons. They are denoted as: eeee, eee $\gamma$, ee $\mu \mu$, e $\gamma \mu \mu$, ee $\tau \tau, \mu \mu \mu \mu, \mu \mu \mu-$ MIP, $\mu \mu \tau \tau$, and $\tau \tau \tau \tau$. Each analysis is used in the study of one or more $h_{\ell \ell^{\prime}}$ couplings, as summarised in Table 2.

In addition, three semi-inclusive selections are devised to increase the selection efficiency for final states with tau leptons decaying into hadrons. These selections first identify an electron or a muon pair in hadronic events, including the case in which one of the electrons is tagged as a photon, and then force the remaining particles of the event into two jets by means of the DURHAM [23] algorithm. These two jets are considered as tau lepton candidates. The selections are denoted as: ee-jet-jet, e $\gamma$-jet-jet and $\mu \mu$-jet-jet. They are used for the analyses of the $h_{\mathrm{e} \tau}, h_{\mu \tau}$ and $h_{\tau \tau}$ couplings, as detailed in Table 2.

\section{Event selection}

Low-multiplicity events with more than three but less than ten tracks and visible energy in excess of $0.3 \sqrt{s}$ are selected. Two classes of events are accepted: events with at least three particles identified as electrons, muons or tau leptons or events with two jets and an electron or muon pair or one electron and one photon. The numbers of events obtained by this preselection are given in Table 3, where the results of the twelve different analyses are combined and presented for the six $h_{\ell \ell^{\prime}}$ couplings. Good agreement is observed between data and Standard Model expectations.

Several discriminating variables are considered to increase the sensitivity of the analysis.

- The energy of the most energetic lepton, $E_{1}$, is close to $0.5 \sqrt{s}$ for the background from two-fermion events, and peaks around $0.25 \sqrt{s}$ for the signal, which predicts a similar energy sharing for all leptons of the event. A cut around $E_{1}<0.45 \sqrt{s}$ is used by all twelve selections. As an example, the distributions for the eee $\gamma$ analysis are shown in Fig. 2(a).

- The energy of the second most energetic lepton tends to be high for background events and peaked 
Table 3

Numbers of events observed in data, $N_{D}$, expected from Standard Model processes, $N_{B}$, and for a $m_{\mathrm{H}}=95 \mathrm{GeV}$ signal, $N_{S}$, after the application of the preselection and final selection cuts. Final selection efficiencies, $\varepsilon$, for $m_{\mathrm{H}}=60-100 \mathrm{GeV}$ are also given

\begin{tabular}{|c|c|c|c|c|c|c|c|}
\hline \multirow[t]{2}{*}{ Coupling } & \multicolumn{3}{|c|}{ Preselection } & \multicolumn{4}{|c|}{ Final results } \\
\hline & $N_{D}$ & $N_{B}$ & $N_{S}$ & $N_{D}$ & $N_{B}$ & $N_{S}$ & $\varepsilon(\%)$ \\
\hline$h_{\mathrm{ee}}$ & 7 & 10.9 & 18.3 & 0 & 2.7 & 16.9 & $46-63$ \\
\hline$h_{\mathrm{e} \mu}$ & 12 & 10.2 & 12.9 & 9 & 6.5 & 12.4 & $35-44$ \\
\hline$h_{\mathrm{e} \tau}$ & 1308 & 1250 & 7.5 & 23 & 21.9 & 6.5 & $39-44$ \\
\hline$h_{\mu \mu}$ & 0 & 1.0 & 10.6 & 0 & 0.7 & 9.2 & $28-32$ \\
\hline$h_{\mu \tau}$ & 8 & 4.4 & 8.2 & 3 & 4.3 & 4.7 & 19-22 \\
\hline$h_{\tau \tau}$ & 1318 & 1258 & 12.5 & 28 & 27.1 & 11.1 & $46-53$ \\
\hline
\end{tabular}
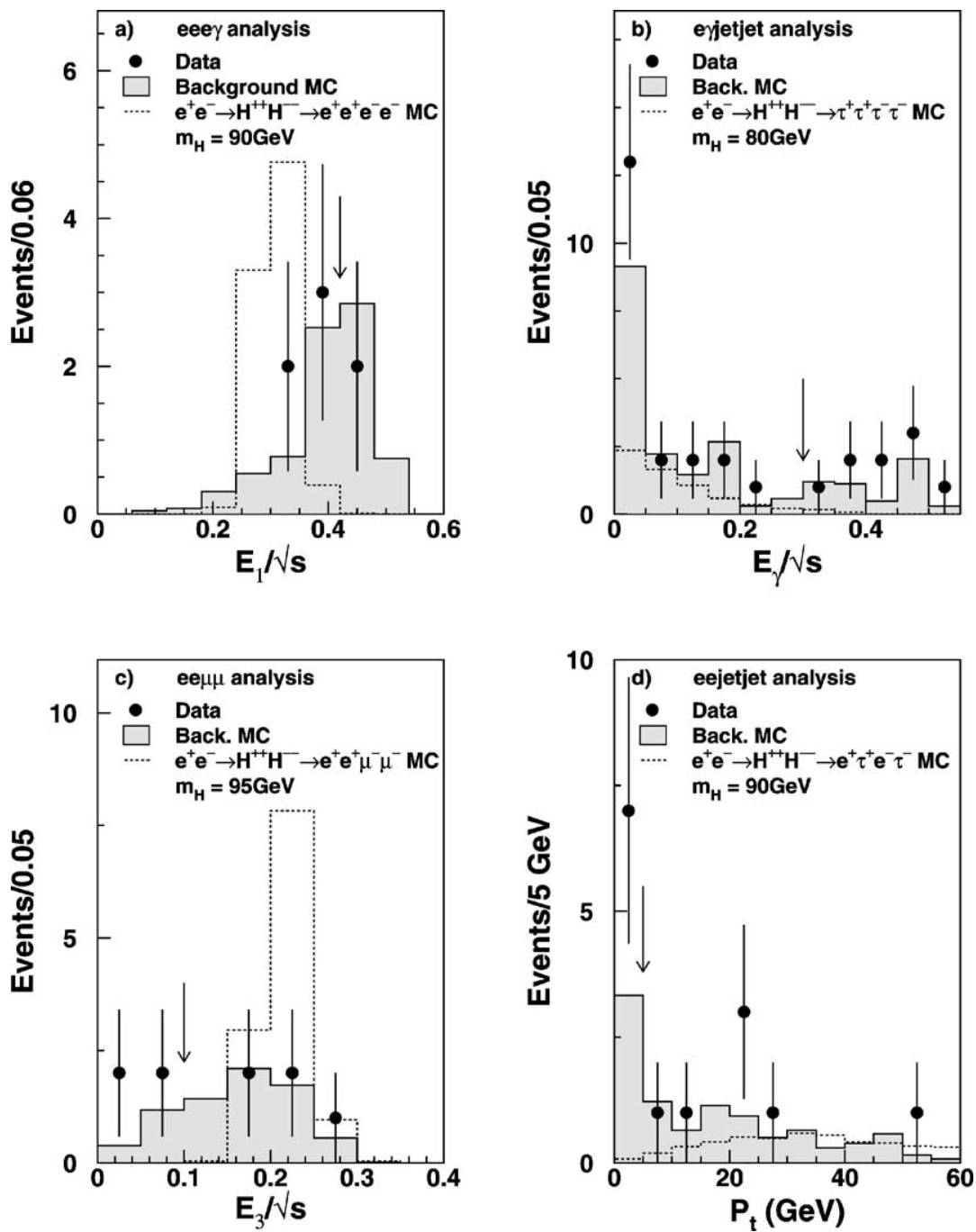

Fig. 2. Distributions for data, signal and background Monte Carlo of: (a) the energy of the most energetic lepton in the eee $\gamma$ analysis, (b) the photon energy for the e $\gamma$-jet-jet analysis, (c) the energy of the third most energetic lepton for the ee $\mu \mu$ analysis and (d) the event transverse momentum for the ee-jet-jet analysis. The arrows indicate the position of the cuts. 
around $0.25 \sqrt{s}$ for the signal. A cut around $0.35 \sqrt{s}$ is applied for the ee-jet-jet and $\mu \mu$-jet-jet analyses.

- The energy of the selected photon, $E_{\gamma}$, for initial state radiation photons from fermion pair-production has a high energy tail, as shown in Fig. 2(b) for the e $\gamma-$ jet-jet selection. A cut around $E_{\gamma}<0.3 \sqrt{s}$ is applied for all analyses which accept photons. For events of the eee $\gamma$ and $\mathrm{e} \gamma \mu \mu$ analyses, an additional cut around $E_{\gamma}>0.2 \sqrt{s}$ is applied, to enforce the signal topology which predicts lepton energies around $0.25 \sqrt{s}$.

- The energy of the third most energetic lepton, $E_{3}$, is low for the background from two-fermion processes and non-resonant or single-resonant fourfermion production and also peaks around $0.25 \sqrt{s}$ for the signal. A cut around $E_{3}>0.1 \sqrt{s}$ is applied for the ee $\mu \mu$ selection, whose distributions are shown in Fig. 2(c).

- Events with jets in the final state suffer from a potentially large background from two-photon processes. This is reduced by requiring that an energy less than $30 \mathrm{GeV}$ is deposited in the calorimeters in a $30^{\circ}$ angle around the beam line and the projection of the missing momentum vector on this direction is less than $50 \mathrm{GeV}$. The presence of neutrinos in tau lepton decays gives signal events a momentum imbalance in the plane transverse to the beam axis, $P_{t}$, as shown in Fig. 2(d) for the ee-jet-jet analysis. A cut $P_{t}>5 \mathrm{GeV}$ is applied, further reducing events from fermion pair-production and two-photon processes which have small values of $P_{t}$.

The twelve selections listed in Table 2 are simultaneously applied and their yields are combined for the six couplings. The nine selections without jets in the final state are largely complementary, while a large overlap is observed between the ee-jet-jet and ee $\tau \tau$ selections. Additional selections like ee $\gamma \gamma$ and $\mu-$ MIP-jet-jet are found not to increase the signal sensitivity.

\section{Results and interpretation}

Table 3 compares the number of events observed after final selection with the Standard Model expectations. Good agreement is observed and no evidence is found for a signal due to doubly-charged Higgs bosons. The number of expected signal events for
$m_{\mathrm{H}}=95 \mathrm{GeV}$ and the selection efficiencies for the range $m_{\mathrm{H}}=60-100 \mathrm{GeV}$ are also given.

The sensitivity of the analysis is enhanced by the reconstruction of the mass of the candidate Higgs bosons. For each coupling, all pairings of leptons with a flavour consistent with doubly-charged Higgs boson decay are considered and their invariant and recoil masses are calculated. The pairing with the smallest difference between these two masses is retained and their average is used as an estimate of $m_{\mathrm{H}}$. The distributions of the reconstructed mass for data, Standard Model and signal Monte Carlo are presented in Fig. 3. No structure possibly due to a doubly-charged Higgs signal is observed.

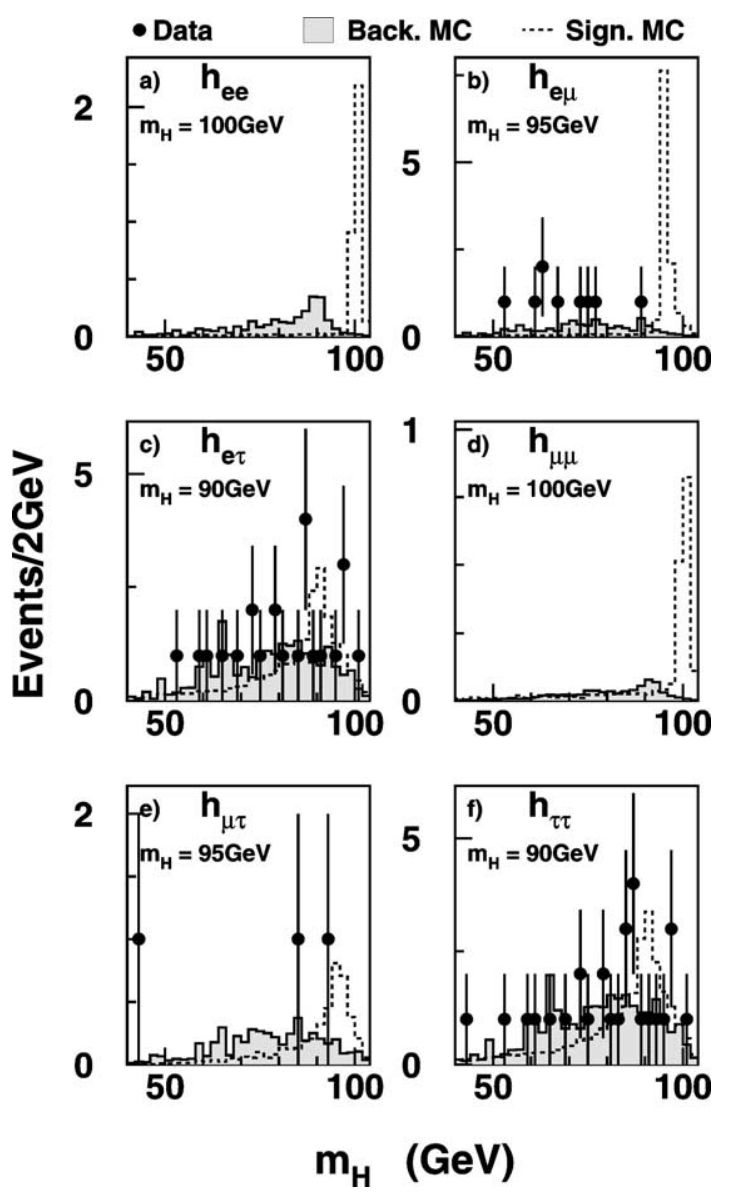

Fig. 3. Distributions for data, signal and background Monte Carlo of the reconstructed Higgs mass for the: (a) $h_{\mathrm{ee}}$, (b) $h_{\mathrm{e} \mu}$, (c) $h_{\mathrm{e} \tau}$, (d) $h_{\mu \mu}$, (e) $h_{\mu \tau}$ and (f) $h_{\tau \tau}$ couplings. 

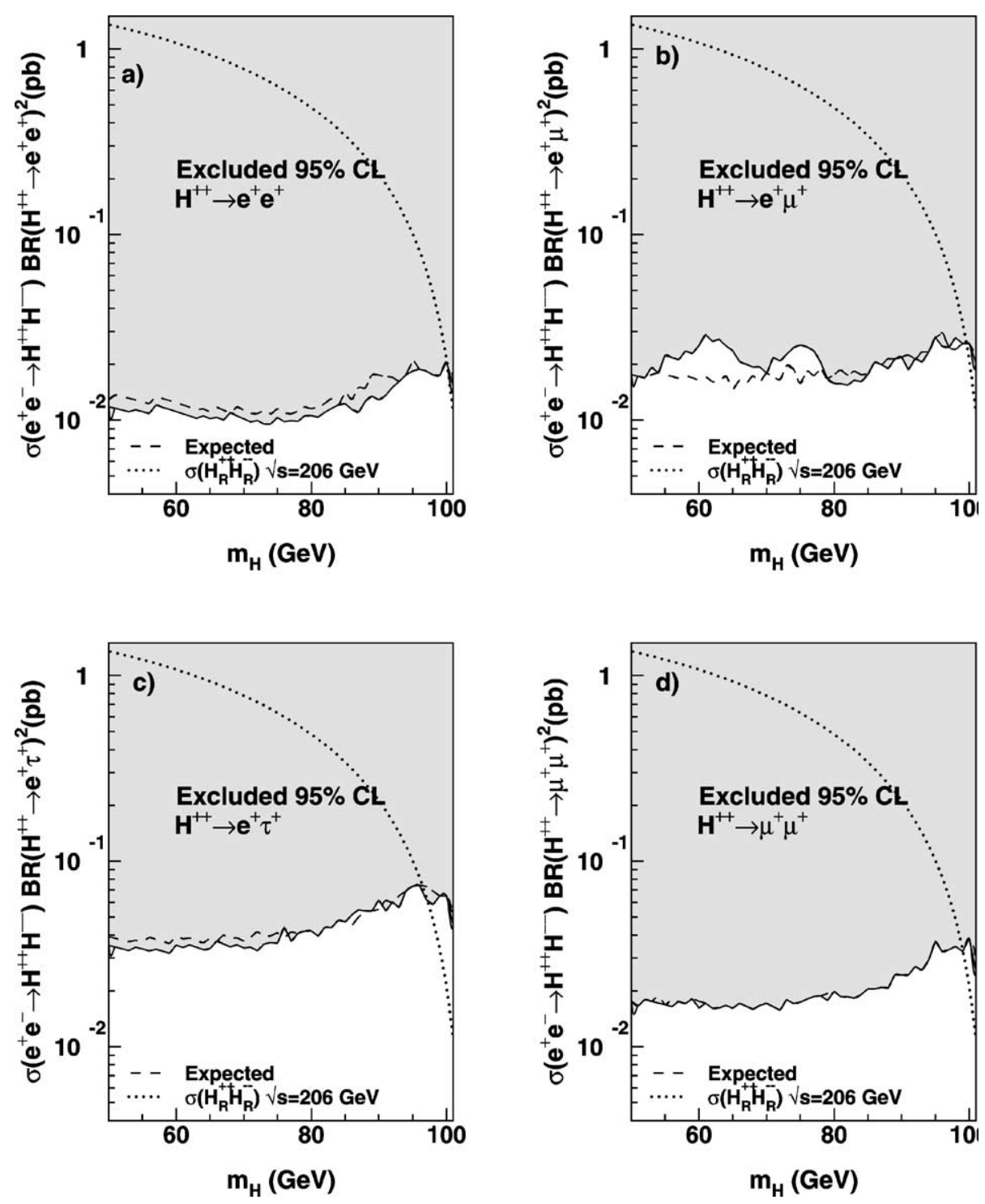

Fig. 4. Observed and expected limits on the cross section of doubly-charged Higgs boson pair-production times its branching ratio in a given final state as a function of $m_{\mathrm{H}}$ for the: (a) $h_{\mathrm{ee}}$, (b) $h_{\mathrm{e} \mu}$, (c) $h_{\mathrm{e} \tau}$ and (d) $h_{\mu \mu}$ couplings. The expected cross section for the $s$-channel production of a right-handed doubly-charged Higgs boson is also shown.

In the absence of a signal, upper limits on the production cross section of doubly-charged Higgs bosons are derived as a function of $m_{\mathrm{H}}$ and converted to lower limits on $m_{\mathrm{H}}$. The log-likelihood ratio technique [4] is used to calculate the observed and expected $95 \%$ confidence level cross section limits, presented, as a function of $m_{\mathrm{H}}$ for the different couplings, in Figs. 4 and 5. Cross sections between 0.1 and $0.01 \mathrm{pb}$ are excluded, depending on $m_{\mathrm{H}}$ and on the coupling.

The limits include systematic uncertainties on the signal efficiency and the background normalisation. These follow from uncertainties in the determination of the energy scale of the detector, on the event selection and lepton identification criteria, on Monte Carlo statistics and on the cross section of the Standard 

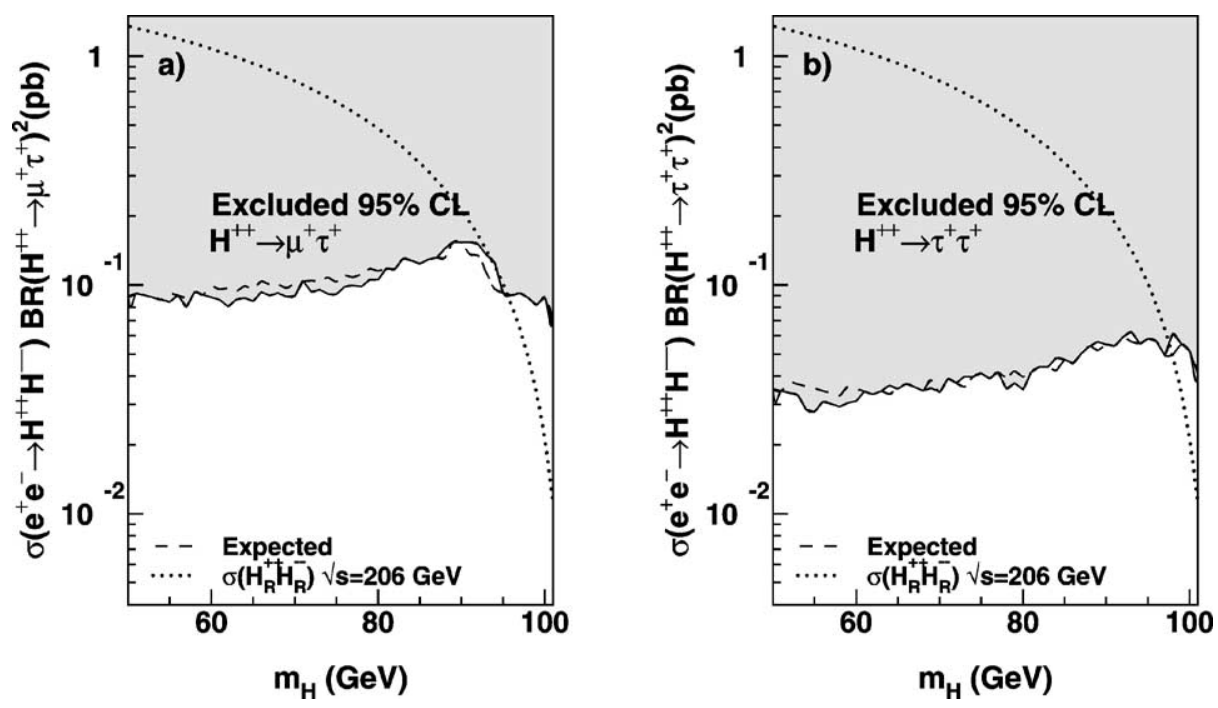

Fig. 5. Observed and expected limits on the cross section of doubly-charged Higgs boson pair-production times its branching ratio in a given final state as a function of $m_{\mathrm{H}}$ for the: (a) $h_{\mu \tau}$ and (b) $h_{\tau \tau}$ couplings. The expected cross section for the $s$-channel production of a right-handed doubly-charged Higgs boson is also shown.

Table 4

Systematic uncertainties on the signal efficiencies and on the background levels for the different couplings

\begin{tabular}{lcc}
\hline Coupling & Signal (\%) & Background (\%) \\
\hline$h_{\mathrm{ee}}$ & 1.8 & 16.8 \\
$h_{\mathrm{e} \mu}$ & 1.8 & 14.5 \\
$h_{\mathrm{e} \tau}$ & 1.8 & 9.3 \\
$h_{\mu \mu}$ & 1.8 & 15.1 \\
$h_{\mu \tau}$ & 1.4 & 10.7 \\
$h_{\tau \tau}$ & 3.2 & 10.4 \\
\hline
\end{tabular}

Table 5

Observed and expected limits on $m_{\mathrm{H}}$ at $95 \%$ confidence level

\begin{tabular}{lcc}
\hline Coupling & Observed $(\mathrm{GeV})$ & Expected $(\mathrm{GeV})$ \\
\hline$h_{\mathrm{ee}}$ & 100.2 & 100.1 \\
$h_{\mathrm{e} \mu}$ & 99.8 & 99.7 \\
$h_{\mathrm{e} \tau}$ & 97.2 & 95.5 \\
$h_{\mu \mu}$ & 99.4 & 99.1 \\
$h_{\mu \tau}$ & 95.5 & 93.8 \\
$h_{\tau \tau}$ & 97.3 & 97.6 \\
\hline
\end{tabular}

Model background processes. Table 4 gives the total systematic uncertainties for the different couplings. These uncertainties reduce the sensitivity by a few hundred $\mathrm{MeV}$.

Lower limits on $m_{\mathrm{H}}$ are extracted by comparing the cross section upper limits with the known cross section of the process $\mathrm{e}^{+} \mathrm{e}^{-} \rightarrow \mathrm{H}^{++} \mathrm{H}^{--}[10,11]$. The most conservative scenario of a right-handed $\mathrm{H}^{ \pm \pm}$and the absence of a $t$-channel contribution to $\mathrm{H}^{ \pm \pm}$production is considered. The observed limits vary from $95.5 \mathrm{GeV}$ to $100.2 \mathrm{GeV}$, depending on the coupling and are listed in Table 5 together with the expected ones.

\section{Constraints from Bhabha scattering}

The measurements of the cross sections and forward-backward asymmetries of the $\mathrm{e}^{+} \mathrm{e}^{-} \rightarrow \mathrm{e}^{+} \mathrm{e}^{-}$ process in $243.7 \mathrm{pb}^{-1}$ of data at $\sqrt{s}=130-189 \mathrm{GeV}$ are described in Ref. [24] and found to be in good agreement with the Standard Model predictions [25, 26]. Similar analyses are applied to $446.8 \mathrm{pb}^{-1}$ of data collected at $\sqrt{s}=192-209 \mathrm{GeV}$. The results are also in good agreement with the Standard Model predictions, and show no evidence for the exchange of a doubly-charged Higgs boson.

A fit for $h_{\mathrm{ee}}$ is performed to the measured cross sections and forward-backward asymmetries for $\sqrt{s}=$ 130-209 GeV and several hypotheses on the value of $m_{\mathrm{H}}$. Experimental systematic uncertainties [24] and uncertainties on the Standard Model predictions [27] are taken into account in the fit. Upper limits on $h_{\mathrm{ee}}$ at $95 \%$ confidence level are derived as a function of 


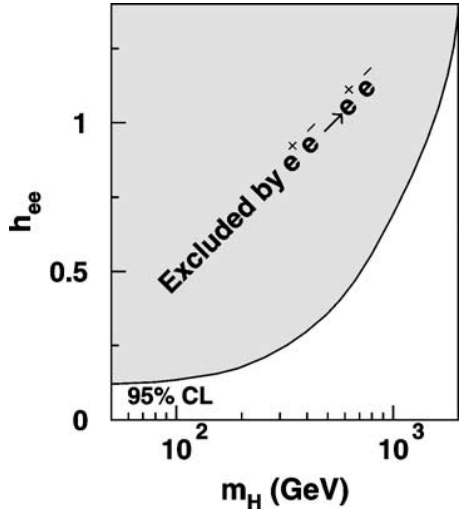

Fig. 6 . Region in the $h_{\mathrm{ee}}$ vs. $m_{\mathrm{H}}$ plane excluded by the study of the $\mathrm{e}^{+} \mathrm{e}^{-} \rightarrow \mathrm{e}^{+} \mathrm{e}^{-}$process.

$m_{\mathrm{H}}$ and shown in Fig. 6. The exclusion region for $h_{\text {ee }}>0.7$ extends to the $\mathrm{TeV}$ scale and is complementary to that derived here from the search for pairproduction of doubly-charged Higgs bosons.

\section{References}

[1] S.L. Glashow, Nucl. Phys. 22 (1961) 579; S. Weinberg, Phys. Rev. Lett. 19 (1967) 1264; A. Salam, in: N. Svartholm (Ed.), Elementary Particle Theory, Almqvist \& Wiksell, Stockholm, 1968, p. 367.

[2] P.W. Higgs, Phys. Lett. 12 (1964) 132; P.W. Higgs, Phys. Rev. Lett. 13 (1964) 508; P.W. Higgs, Phys. Rev. 145 (1966) 1156; F. Englert, R. Brout, Phys. Rev. Lett. 13 (1964) 321; G.S. Guralnik, C.R. Hagen, T.W.B. Kibble, Phys. Rev. Lett. 13 (1964) 585.

[3] L3 Collaboration, P. Achard, et al., Phys. Lett. B 517 (2001) 319.

[4] ALEPH, DELPHI, L3 and OPAL Collaborations, LEP Working Group for Higgs Boson Searches, Phys. Lett. B 565 (2003) 61.

[5] R.N. Mohapatra, J.D. Vergados, Phys. Rev. Lett. 47 (1981) 1713; G.B. Gelmini, M. Roncadelli, Phys. Lett. B 99 (1981) 411; V.D. Barger, et al., Phys. Rev. D 26 (1982) 218; T. Rizzo, Phys. Rev. D 25 (1982) 1355; M. Lusignoli, S. Petrarca, Phys. Lett. B 226 (1989) 397; J.F. Gunion, Int. J. Mod. Phys. A 11 (1996) 1551; R.N. Mohapatra, G. Senjanovic, Phys. Rev. D 23 (1981) 165.

[6] N. Arkani-Hamed, A.G. Cohen, H. Georgi, Phys. Lett. B 513 (2001) 232;

N. Arkani-Hamed, et al., JHEP 0208 (2002) 020;

N. Arkani-Hamed, et al., JHEP 0208 (2002) 021;

T. Han, et al., Phys. Rev. D 67 (2003) 0905004.

[7] C.S. Aulakh, et al., Phys. Rev. D 57 (1998) 4174; Z. Chacko, R.N. Mohapatra, Phys. Rev. D 58 (1998) 015003;
B. Dutta, R.N. Mohapatra, Phys. Rev. D 59 (1999) 015018.

[8] L3 Collaboration, B. Adeva, et al., Nucl. Instrum. Methods A 289 (1990) 35;

L3 Collaboration, O. Adriani, et al., Phys. Rep. 236 (1993) 1; M. Chemarin, et al., Nucl. Instrum. Methods A 349 (1994) 345; G. Basti, et al., Nucl. Instrum. Methods A 374 (1996) 293; A. Adam, et al., Nucl. Instrum. Methods A 383 (1996) 342; I.C. Brock, et al., Nucl. Instrum. Methods A 381 (1996) 236; M. Acciarri, et al., Nucl. Instrum. Methods A 351 (1994) 300.

[9] OPAL Collaboration, G. Abbiendi, et al., Phys. Lett. B 526 (2002) 221;

DELPHI Collaboration, J. Abdallah, et al., Phys. Lett. B 552 (2003) 127; OPAL Collaboration, G. Abbiendi, et al., hep-ex/0308052.

[10] M.L. Swartz, Phys. Rev. D 40 (1989) 1521.

[11] K. Huitu, et al., Helsinki Institute of Physics Report HIP-199806 (1998).

[12] F.A. Berends, R. Kleiss, Nucl. Phys. B 260 (1985) 32.

[13] PHOTOS version 2.0 is used; E. Barberio, Z. Wạs, Comput. Phys. Commun. 79 (1994) 291.

[14] TAUOLA version 2.4 is used; S. Jadach, et al., Comput. Phys. Commun. 76 (1993) 361.

[15] JETSET version 7.3 and PYTHIA version 5.722 are used; T. Sjöstrand, preprint CERN-TH/7112/93 (1993), revised 1995;

T. Sjöstrand, Comput. Phys. Commun. 82 (1994) 74.

[16] KK2f version 4.14 is used;

S. Jadach, B.F.L. Ward, Z. Wạs, Comput. Phys. Commun. 130 (2000) 260.

[17] BHWIDE version 1.03 is used;

S. Jadach, W. Placzek, B.F.L. Ward, Phys. Lett. B 390 (1997) 298.

[18] EXCALIBUR version 1.11 is used;

F.A. Berends, R. Kleiss, R. Pittau, Comput. Phys. Commun. 85 (1995) 437.

[19] KORALW version 1.33 is used;

M. Skrzypek, et al., Comput. Phys. Commun. 94 (1996) 216;

M. Skrzypek, et al., Phys. Lett. B 372 (1996) 289.

[20] PHOJET version 1.05 is used;

R. Engel, Z. Phys. C 66 (1995) 203;

R. Engel, J. Ranft, Phys. Rev. D 54 (1996) 4244.

[21] DIAG 36 Monte Carlo;

F.A. Berends, P.H. Daverfeldt, R. Kleiss, Nucl. Phys. B 253 (1985) 441.

[22] GEANT version 3.15 is used;

R. Brun, et al., preprint CERN DD/EE/84-1 (1985), revised 1987;

The GHEISHA program (H. Fesefeldt, RWTH Aachen Report PITHA $85 / 02,1985$ ) is used to simulate hadronic interactions.

[23] S. Bethke, et al., Nucl. Phys. B 370 (1992) 310.

[24] L3 Collaboration, M. Acciarri, et al., Phys. Lett. B 479 (2000) 101.

[25] ZFITTER version 6.21 is used; D. Bardin, et al., Comput. Phys. Commun. 133 (2001) 229.

[26] TOPAZO version 4.4 is used; G. Montagna, et al., Comput. Phys. Commun. 76 (1993) 328.

[27] M. Kobel, et al., CERN Report 2000-009, hep-ph/0007180. 